\title{
Objective Function Migration with Wavepath
}

Yike Liu ${ }^{1 *}, \mathrm{Xu}$ Chang ${ }^{1}$, Hongchuan $\mathrm{Sun}^{2}$,

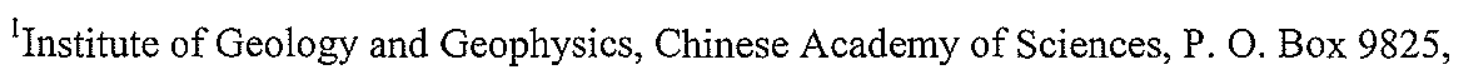
Beijing 100029, China

${ }^{2}$ Department of Geology and Geophysics, University of Utah, 717 WBB, 135 South 1460

East, Salt Lake City, UT 84112, USA

*E-mail: ykliu@mail.igcas.ac.cn

\begin{abstract}
We present a new least-squares migration method called least-squares wavepath migration (LSWM) or Objective Function Migration with Wavepath. The proposed method combines an iterative conjugate gradient solver with a stationary-phase wavepath migration operator. Numerical tests demonstrate that (a) LSWM is computationally more efficient than and almost as accurate as Kirchhoff least-squares migration, and (b) many of the artifacts seen in wavepath migration images are suppressed after several conjugate gradient iterations. Previous results showed that 3-D wavepath migration is up to 100 times as fast as a standard 3-D Kirchhoff migration, but sometimes at the cost of reduced quality. With the proposed LSWM method, the image quality in wavepath migration can be improved at an acceptable increase in computational cost.
\end{abstract}

\section{INTODUCTION}

The objective of this paper is to combine least-squares migration and wavepath migration and to form a so-called least-squares wavepath migration (LSWM). Least-squares migration (LSM) is capable of suppressing migration artifacts and 
producing better images compared to standard migration methods. However, it is computationally much more expensive as the data are migrated and modelled in an iterative way. To reduce the computational burden of LSM, we resort to wavepath migration (WM), a stationary-phase migration method that can be up to orders of magnitude faster than a standard Kirchhoff migration (KM). With WM replacing KM as the modelling and migration operator, LSWM can be considerably faster than a standard Kirchhoff LSM. On the other hand, the determination of wavepath in WM is sensitive to the data recording geometry and the signal-to-noise ratio, which could lead to compromised imaging quality. With the data being migrated in a least-squares way, the migration artifacts in WM can be effectively suppressed. Thus, the combined method, LSWM, will be expected to achieve a better trade-off of computational efficiency and imaging quality (Liu 2005).

Least-squares migration (LSM) finds the reflectivity distribution that minimizes the squared error between the predicted and observed seismograms (Nemeth et al. 1999; Duquet et al. 2000):

$$
\varepsilon=1 / 2\|L m-d\|^{2}
$$

which yields the least-squares solution:

$$
m=\left[L^{T} L\right]^{-1} L^{T} d
$$

Here $d$ is the difference between the predicted traces due to a smooth background velocity model and the observed data, the operator $L$ represents Born forward modelling using the migration velocity model, and $m$ is the perturbed slowness referred to as the reflectivity distribution.

In comparison, standard migration (Claerbout 1992; Stolt and Benson 1986) smears each time sample along a quasi-ellipsoid in the reflectivity model and sometimes weights the $i$ th reflectivity cell by $1 /\left[L^{T} L\right]_{i i}$; which is a crude approximation to the inverse Hessian in equation (2). The primary motivation for using LSM is that it can significantly reduce migration artifacts due to trace gaps, irregular source and receiver sampling and uneven illumination in the subsurface due to a complex velocity model (Nemeth 1999). 
The problem with LSM is that it is more than an order of magnitude more expensive than standard migration (Cole and Karrenbach 1992). This is because a conjugate gradient method iteratively migrates and forward models the data to minimize the data residual in equation (1). In practice, ten or more iterations are needed for useful results, a cost that is still too expensive for routine data imaging.

To significantly reduce the computational expense of LSM we propose to replace its migration $L^{T}$ and modelling $L$ operators by a stationary-phase approximation to the Kirchhoff integral, denoted as wavepath migration (Sun and Schuster 2000). We shall denote the combination of using least-squares with wavepath migration as least-squares wavepath migration (LSWM). Briefly, wavepath migration smears a trace's energy along fat rays or wavepaths rather than a volume of quasi-concentric ellipsoids. The width of the fat ray or wavepath is proportional to the wavelength, and the intersection of the source and the receiver wavepaths defines the specular Fresnel zone from which the reflection originated (see Figure 1). The dimension of a fat ray is about 1.5-D, so it is fewer dimensions than a 3-D volume of ellipsoids. The dimension of a fat ray is roughly $1.5 \mathrm{D}$ in a velocity model with volume $\mathrm{N}^{\wedge} 3$. By this we mean that there is one very long axis along the central ray that typically has a length that is on the order of $\mathrm{N}$; as the model size grows so does the length of the wavepath. On the other hand, the maximum width of the wavepath is proportional to the square root of the raypath's length (Schuster, 1996); thus, the volume of the wavepath is on the order of $\mathrm{N}^{\wedge} 3 / 2$, or $1.5 \mathrm{D}$. Thus, the computational cost of $\mathrm{WM}$ is, in principle, considerably less than that of KM, and can be orders of magnitude less expensive (Sun and Schuster 2001). Therefore, the proposed LSWM method can be significantly faster than a standard Kirchhoff LSM. 


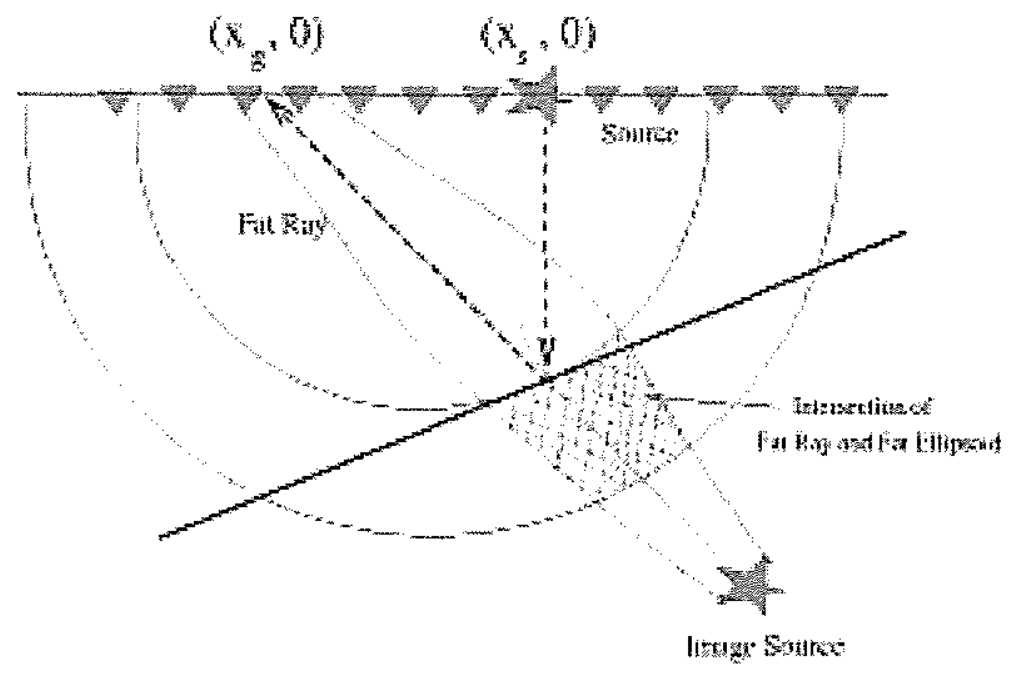

Figure 1 Dipping reflector embedded in a homogeneous half space below a receiver array. The usual $\mathrm{KM}$ algorithm smears reflection energy along the fat ellipsoid. The intersection of the fat ray and the fat ellipsoid is where the reflection energy is smeared by LSWM.

\section{THEORY}

Assuming geophones on the surface at $\left(x_{g}, 0\right)$, and a buried single reflector such that a high-frequency common-shot gather of trace $\tilde{D}\left(x_{g}, 0\right)_{w}$ for a harmonic point source at $\left(x_{s}, 0\right)$ is expressed as

$$
\widetilde{D}\left(x_{g}, 0\right)_{\omega}=\tilde{W}(\omega) A\left(x_{s}, x_{g}\right) e^{i \omega \tau s g}
$$

where contains geometric spreading information, is the spectrum of the source wavelet, and is the reflection traveltime for primary reflections from a single subsurface reflector(see Figure 1)

The prestack migrated section can be computed by substituting equation (3) into the diffraction stack formula:

$$
\begin{aligned}
& \tilde{M}(r, \omega)=\int_{0}^{L} \widetilde{D}\left(x_{g}, 0\right)_{\omega} B\left(x_{s}, r, x_{g}\right) e^{-i \omega(\tau g r+\tau s r)} d x_{g} \\
& =\int_{0}^{L} \tilde{W}(\omega) A\left(x_{s}, x_{g}\right) B\left(x_{s}, r, x_{g}\right) \times e^{-i \omega(\tau g r+\tau s r-\tau s g)} d x_{g}
\end{aligned}
$$


where $B\left(x_{s}, r, x_{g}\right)$ contains geometric spreading terms, and $\tau_{g r}$ and $\tau_{s r}$ correspond to the traveltimes for energy to propagate from the geophone to the subsurface interrogation point at $r$ and from the source to the interrogation point, respectively.

Defining $\phi\left(x_{g}\right)=\tau_{s g}-\tau_{g r}-\tau_{s r}$ where the $x_{s}$ and $r$ terms are suppressed, setting $C\left(x_{g}, r, x_{s}\right)=A\left(x_{s}, x_{g}\right) B\left(x_{s}, r, x_{g}\right)$ in equation (4), and assuming a sufficiently high source frequency yields the stationary phase approximation to the migration equation:

$$
\begin{aligned}
& \tilde{M}(r, \omega)=\tilde{W}(\omega) \int_{b}^{L} C\left(x_{g}, r, x_{s}\right) e^{i \omega \phi\left(x_{g}\right)} d x_{g} \\
& \sim e^{i \omega \phi\left(x_{g}^{*}\right)+i \mu \pi / 4} \tilde{W}(\omega) C\left(x_{g}^{*}, r, x_{s}\right) \sqrt{\frac{2 \pi}{\omega\left|\phi\left(x_{g}^{*}\right)^{\prime \prime}\right|}}
\end{aligned}
$$

where $x_{g}^{*}$ is the stationary point of $\phi\left(x_{g}\right), \mu=\operatorname{sign}\left(\phi\left(x_{g}^{*}\right)^{\prime \prime}\right)$, , and $\phi\left(x_{g}\right)^{\prime \prime}$ is the second derivative of $\phi$ with respect to $x_{g}$. Here, we assume a first-order stationary point that is interior to the integration range. The formula for the case of and exterior stationary point is given in Bleistein (1984).

Integrating equation (3) over all frequencies results in the formula:

$$
m(r)=\left.w_{\text {mod }}\left(\tau_{s g}-\tau_{g r}-\tau_{s r}\right)\right|_{r \in \text { raypath }}
$$

where

$$
w_{\text {mod }}(t)=2 \pi C\left(x_{g}^{*}, r, x_{s}\right)\left[1 / \sqrt{t} * \int_{-\infty}^{\infty} \tilde{W}(\omega) e^{i \omega t+i \mu \pi / 4} d \omega\right] / \sqrt{\mid \phi\left(x_{g}^{*}\right)^{\prime \prime}}
$$

and $*$ denotes temporal convolution (Sun and Schuster, 2001).

Equation (6) says that, for the trace at $\left(x_{g}, 0\right)$, the reflection amplitude at time $\tau_{s g}$ should be relocated to the specular reflection point. This compares to a typical migration formula for a single trace, 


$$
m(r)=\left.w\left(\tau_{s g}-\tau_{g r}-\tau_{s r}\right)\right|_{r \in \text { model.volume }}
$$

Which says that, for the single trace, the reflection amplitude at time $\tau_{s g}$ should be smeared in model space within the fat ellipsoid shown in Figure 1. Here, and ellipse is described by $\delta\left(\tau_{s g}-\tau_{g r}-\tau_{s r}\right)$, and $\mathrm{w}(\mathrm{t})$ is the source wavelet function.

Equation (6) can be symbolically re-expressed as

$$
m(r)=w_{\bmod }\left(\tau_{s g}-\tau_{g r}-\tau_{s r}\right) \delta\left(\tau_{g r}+\tau_{s^{*} r}-\tau_{s g}\right)
$$

where $\tau_{s i r}$ is the traveltime for energy to propagate from the image source point(denoted as $\left.s^{*}\right)$ to the interrogation point at $r$. Thus $\delta\left(\tau_{g r}+\tau_{s^{*} r}-\tau_{s g}\right)$ describe the direct ray that starts as solid line from the image source in Figure 1.

The theory of wavepath migration can be summarized as following three steps:

1) Apply a local slant stack to traces in a shot gather, where the central trace at receiver point $r$ is the starting point for the receiver ray. The slant angle that maximizes the coherency in the slant stack and the near-surface velocity are used to determine the angle of incidence of the receiver ray. The shot point is at point $s$.

2) The data are resorted to common receiver gathers with the new shot point at $g$ and the new central receiver point at $s$. The data are slant stacked at the new central receiver $s$ to give the slant angle with maximum coherency. This slant angle is used to determine the source ray at $s$. In practice, the number of traces used for slant stacking is the same number of traces within a Fresnel area of the data, usually 3-7 traces.

3) Wavepaths are constructed along both the receiver and source rays, and their intersection point defines the Fresnel area of the specular reflection point. The width of the fat ray or wavepath is proportional to the wavelength, and the slant-stacked reflection amplitude is smeared along this Fresnel zone. The above procedure is repeated for every third or fourth trace in a shot gather; the skipped traces do not need to be migrated because their reflection information is embedded in the slant stacked 
trace.

If $d$ is the length of the model cube (see Figure 1), the KM operator for one trace occupies a $d^{3}$ volume of overlapping concentric quasi-ellipsoids, compared to the effective $d^{1.5}$ volume occupied by a wavepath of the 3-D WM migration operator. The WM section is sometimes plagued by weak reflectivity. This is partly because the reflection event is smeared along a small Fresnel zone rather than a rather large fat ellipse. Smearing the reflection event along a fat ellipse tends to mitigate problems due to inaccurate migration velocities because there are more opportunities for large ellipses to constructively intersect. In contrast, there are fewer of these constructive opportunities when events are smeared along a small Fresnel zone as in WM, so that dim spots might appear in the section. In addition, LSWM promises to alleviate the recording footprint or migration artifacts due to poor source-receiver geometry.

To overcome the problem of weak reflectivities in the WM image, we propose to formulate the WM equation as a least squares problem, and solve it by a few iterations of the conjugate gradient method. This might be an effective approach because the migration reflectivity model estimated by a conjugate gradient method will be consistent with the data in a least-squares sense. Only strong, not weak, reflectivities in the WM image will be consistent with strong amplitude reflection in the measured data. We will denote this method as least-squares wavepath migration or LSWM.

As the iteration proceeds, these strong reflectivities will be updated to better simulate the observed data. During each iteration, wavepath migration backprojects the residual data followed by the application of the forward modelling operator $L$ to the updated reflectivity model. This forward modelling operator only simulates waves along the pre-determined downgoing wavepath. Reflectivities within the shadowed Fresnel zone exclusively contribute to the forward modelled seismograms.

\section{NUMERICAL EXAMPLES}

The least-squares wavepath migration method is now tested on both synthetic and field data sets. The synthetic data are associated with a point scatterer model, and the field 
data were collected by Mobil from the North Sea.

\section{Point Scatterer Model}

A point scatterer model is used to test the effectiveness of the LSWM method. There are 101 shot stations uniformly distributed along the free surface with a shot interval of $40 \mathrm{~m}$. Each shot gather consists of 201 traces with a geophone interval of $20 \mathrm{~m}$. The source is a $50-\mathrm{Hz}$ Ricker wavelet, the medium velocity is a homogeneous $5000 \mathrm{~m} / \mathrm{s}$, the time sampling interval is $1.0 \mathrm{~ms}$, and the model is represented by a $201 \times 201$ grid with a horizontal and vertical spacing of $20 \mathrm{~m}$. A diffraction stack forward modelling algorithm is used to generate the synthetic seismograms.

Figure 2(a) shows the forward modelled zero-offset data. Figure 2(b) shows the KM image, where the point scatterer is well resolved, but the migration artifacts are also clearly seen. In contrast, the WM image in Figure 2(c) shows much fewer migration artifacts, and the point scatterer is as well resolved as in the KM image. Figure 2(d) shows the LSWM image, which is superior in both image fidelity and artifact attenuation. Ten iterations were used to generate the LSWM image. 


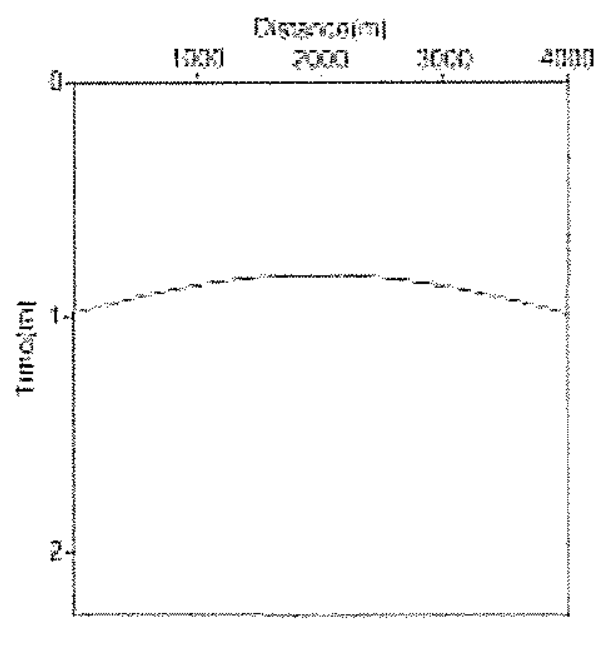

(a)

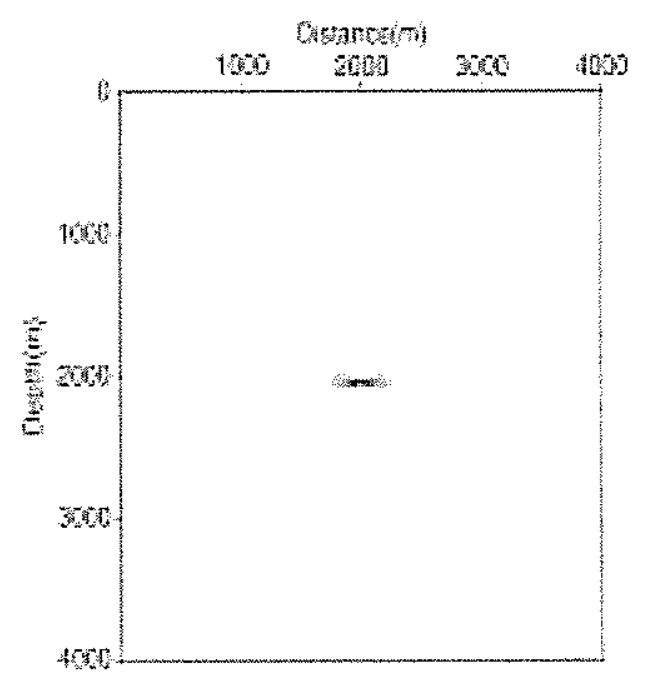

(c)

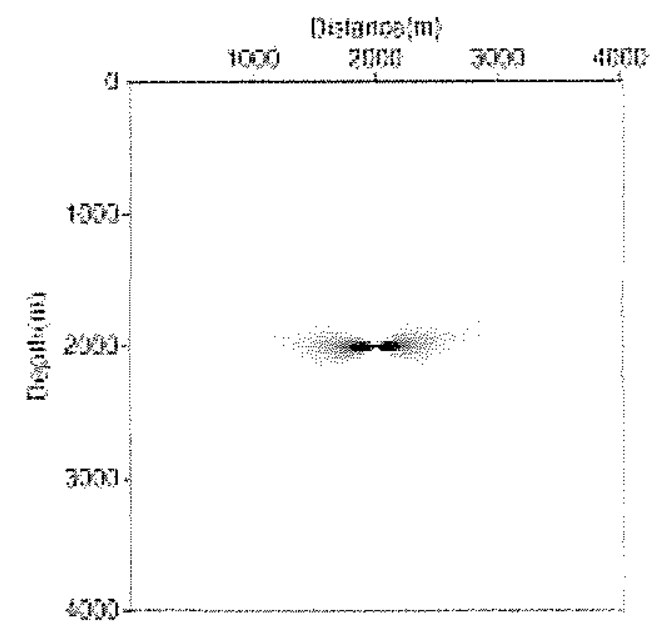

(b)

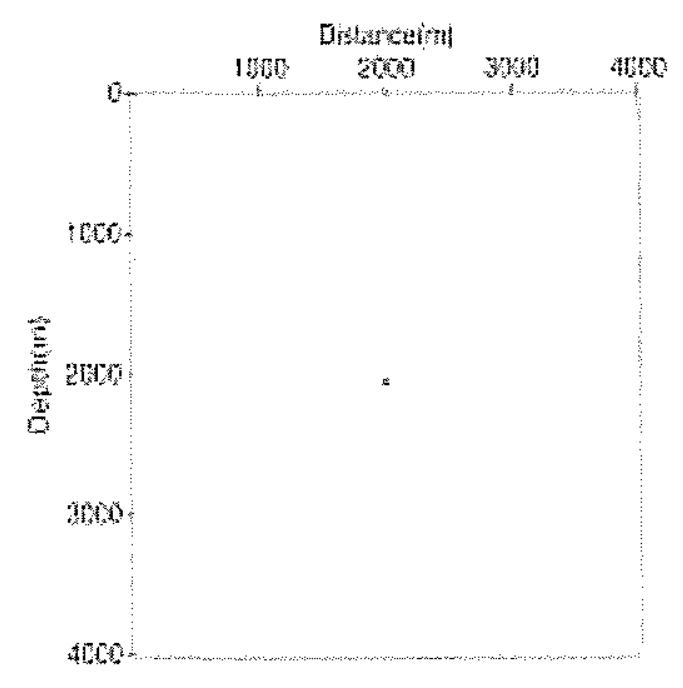

(d)

Figure 2 Synthetic data and migration images: (a) forward modelled zero-offset data, (b) KM image, (c) WM image, and (d) LSWM image. The LSWM image contains the fewest migration artifacts and shows the best image resolution for the point scatterer. 


\section{North Sea Field Data}

The Mobil data were provided courtesy of Robert Keys (ExxonMobil). It is a marine data set, with sources and receivers deployed along a 2-D line. There are 668 shot gathers, each of which contains 120 traces. Both the source and the receiver intervals are $25 \mathrm{~m}$. The recording length is 1500 samples with a time sampling interval of $4 \mathrm{~ms}$. The model consists of a $1594 \times 281$ grid with a grid interval of $12.5 \mathrm{~m}$ in both the horizontal and vertical directions.

Figure 3 shows the WM image (a) and the LSWM image (b) after fifteen iterations. All of the traces were used in the migration. Compared to the WM image, the LSWM image shows better energy focusing, interface continuity, and image resolution. Major layers are more clearly defined in the LSWM image, facilitating geological interpretations.

Figure 4 shows a zoom view of the boxed area in Figure 3. Note that the LSWM image in Figure $4 \mathrm{~b}$ defines the interfaces better and further improves the image resolution. 


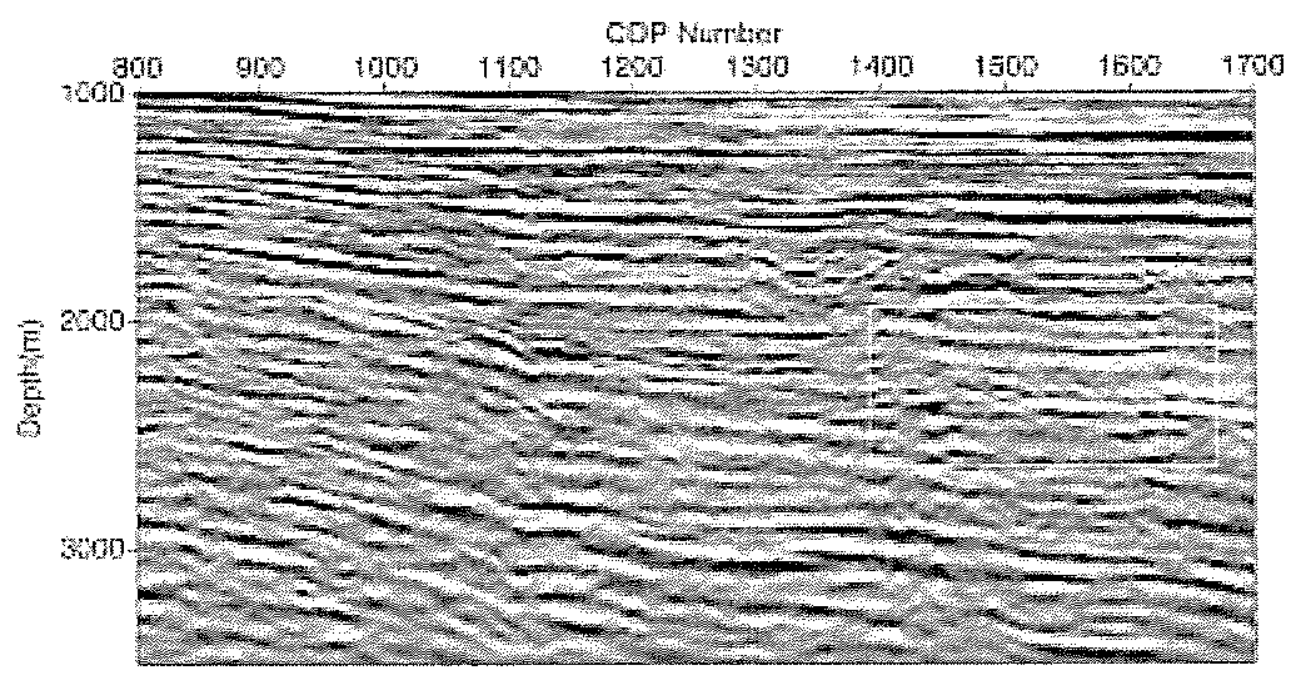

(a)

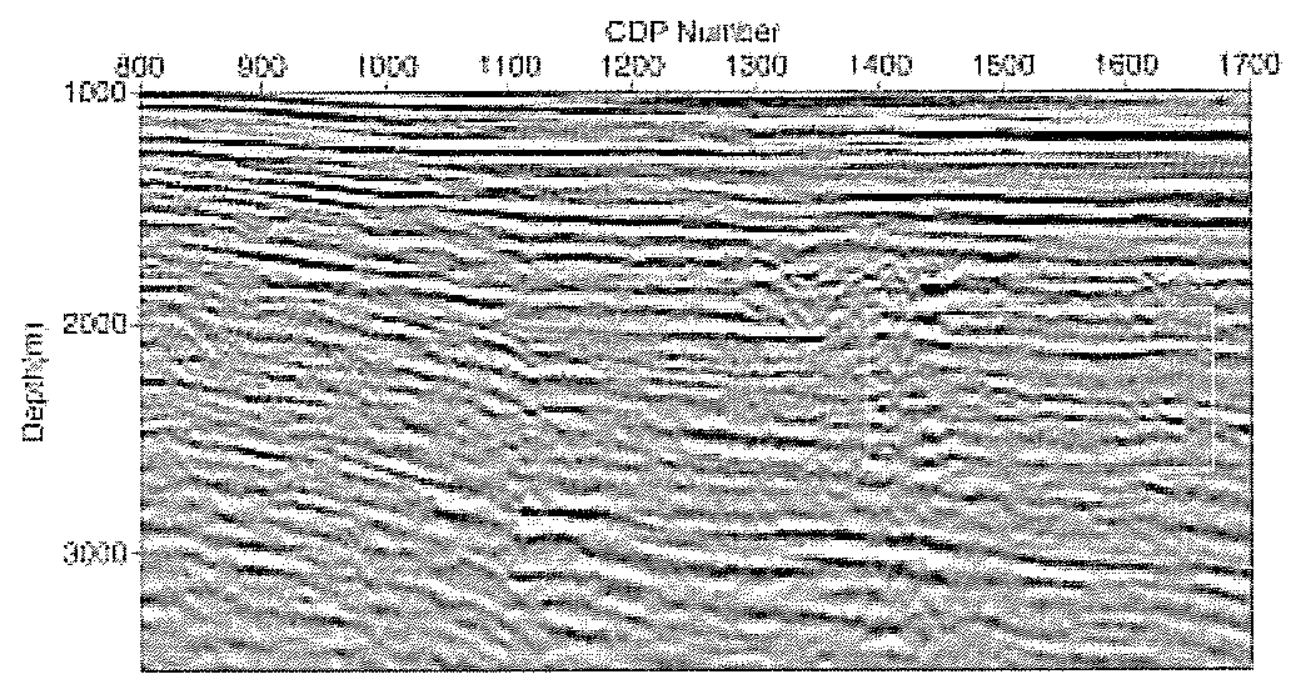

(b)

Figure 3 Comparison of migration images for (a) WM image and (b) LSWM image. The LSWM image shows better energy focusing and interface continuity. Fifteen iterations were used to generate the LSWM image. 


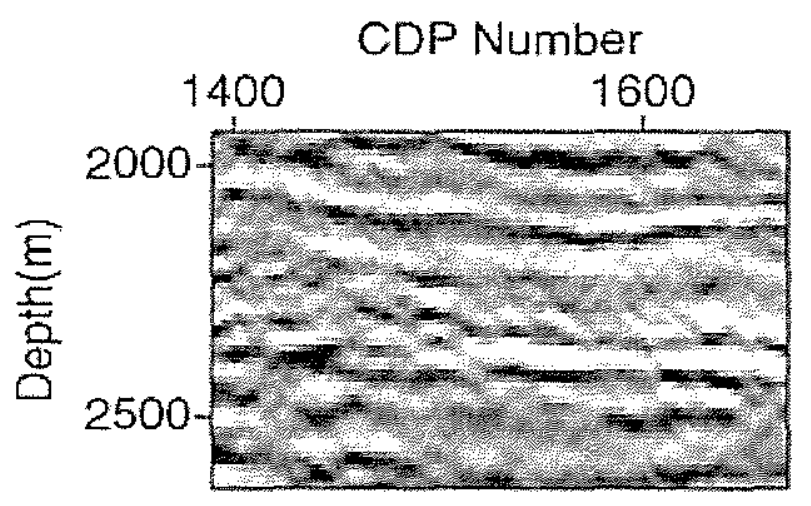

(a)

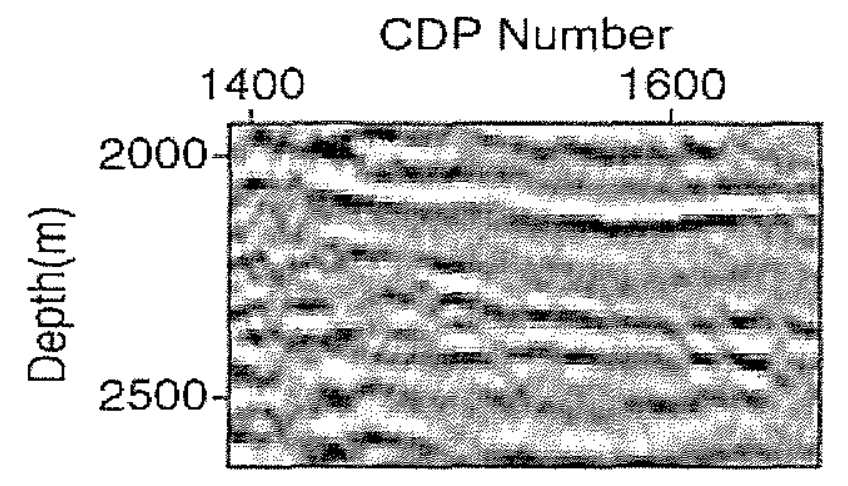

(b)

Figure 4 Zoom view of the boxed area in Figure 3 for (a) WM image and (b) LSWM image. The LSWM image in (b) contains fewer migration artifacts, enhances the reflection interfaces and further improves the image resolution. 


\section{CONCLUSIONS}

We have presented a potentially efficient form of least-squares migration denoted as least-squares wavepath migration. Numerical results with both synthetic and field data show that, compared to wavepath migration, LSWM can effectively reduce migration artifacts and improve image resolution. In addition, energy focusing and interface continuity are also noticeably improved in the LSWM image. Typically, ten to fifteen iterations are required by LSWM to achieve an acceptable residual level.

For the 2-D field data, CPU comparison shows that wavepath migration is 1.5 times faster than standard Kirchhoff migration. Thus, compared to a standard KM method, our LSWM scheme is still much more expensive. For 3-D cases, however, LSWM could require similar or even less computational cost compared to KM. The reason is that 3-D wavepath migration is more than an order-of-magnitude faster than 3-D Kirchhoff migration (Sun and Schuster, 2000). On the other hand, for 3-D migration where severe acquisition footprints usually exist, LSWM could be able to improve the image quality more effectively. Future work will thus apply LSWM to 3-D depth imaging.

\section{ACKNOWLEDGEMENT}

This research was partially funded by the National Natural Science foundation of China (grants numbered 40235055 and 40374050), and the National High Technology Research and Development Program of China (863 Program), we are also grateful for the financial support from the members of the 2001 University of Utah Tomography

\section{REFERENCES}

Bleistein, N., 1984, Mathematical methods for wave phenomena: Academic Press.

Claerbout J. 1992. Earth soundings analysis: processing versus inversion. Black well science Ltd.

Cole S. and Karrenbach M. 1992. Least squares Kirchhoff migration: http://sepwww.stanford.edu/public/docs/75SEP-75.

Duquet B. Marfut K. J. and Dellinger J. A. 2000. Kirchhoff modelling, inversion for 
reflectivity, and subsurface illumination. Geophysics 65,1195-1209.

Liu Y., Sun H. Chang X. and Schuster T., 2005, Least-squares wavepath migration, Geophysical Prospecting, (in press).

Nemeth, T. Wu C. and Schuster G. 1999. Least-squares migration of incomplete reflection data: Geophysics 64, 208-221.

Schuster, G.T., 1996, Resolution limits for crosswell migration and Traveltime tomography: Geophysical J. Int., 427-440.

Slot R. and Benson A. 1986. Seismic migration: Theory and practice: in Handbook of Geophysical Exploration, volume 5, geophysical press, London, UK.

Sun H. and Schuster G. 2000. Wavepath migration versus Kirchhoff migration: 3-D

prestack examples. 70th Ann. Internat. Mtg., Soc. Expl. Geophys., Expanded Abstracts, 977-980.

Sun H. and Schuster G. 2001. 2-D Wavepath migration. Geophysics 66, 1528-1537. 